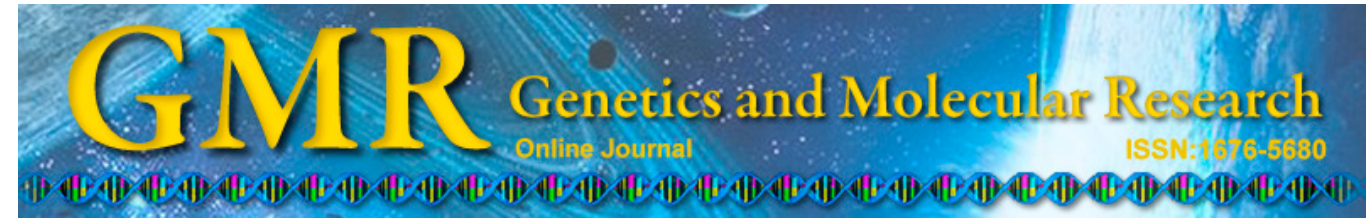

\title{
Effects of conservative laparoscopic endometrial cystectomy and use of kidney-reinforcing and blood activating traditional Chinese medicine on ovarian functions
}

\author{
Y.H. Huang ${ }^{1,2}$, L. Shen ${ }^{2}$, A.H. Cai ${ }^{3}$ and X.F. Liang ${ }^{4}$ \\ 'Department of Gynecology, \\ Wuhan City Hospital of Tradiocional Chinese Medicine, \\ Wuhan, Hubei, China \\ ${ }^{2}$ Department of Traditional Chinese Medicine, Wuhan Union Hospital, \\ Tongji Medical College, Huazhong University of Science and Technology, \\ Wuhan, Hubei, China \\ ${ }^{3}$ Department of Gastroenterology, \\ Guangdong Provincial Hospital of Chinese Medicine, \\ Guangzhou, Guangdong, China \\ ${ }^{4}$ Department of Gynecology, \\ Guangdong Provincial Hospital of Chinese Medicine, \\ Guangzhou, Guangdong, China \\ Corresponding author: L. Shen \\ E-mail: linshencn@126.com
}

Genet. Mol. Res. 14 (1): 645-650 (2015)

Received May 15, 2014

Accepted September 23, 2014

Published January 30, 2015

DOI http://dx.doi.org/10.4238/2015.January.30.6

ABSTRACT. This study investigated functional changes in the ovaries
of women who have had conservative laparoscopic endometrial
cystectomy and the effects of traditional kidney-reinforcing and blood-
activating Traditional Chinese Medicine (TCM) on ovarian function.
Seventy female patients who underwent laparoscopic endometrial
cystectomy were randomized into two groups: TCM group receiving 
kidney-reinforcing and blood-activating Chinese medicine $(\mathrm{N}=35)$ and control group receiving only routine follow-up $(\mathrm{N}=35)$. The serum levels of all study participants were measured for follicle-stimulating hormone (FSH), luteinizing hormone (LH), estradiol (E2) and inhibin $B$ (INHB) prior to and after endometrial surgery. Postoperative menstruation conditions were also assessed. Compared to preoperative conditions, both LH and FSH levels during the postoperative 1st month increased in both groups, while E2 and INHB levels decreased $(\mathrm{P}<0.05)$. In the TCM group, in contrast to the control group and the postoperative 1st month, we observed a decrease in LH and FSH levels during the postoperative 4th month, while E2 and INHB levels increased $(\mathrm{P}<$ 0.05). In this study, we found that certain TCM prescriptions lowered postoperative serum FSH and LH levels and increased the serum INHB and basal E2 levels, thereby improving the ovarian reserve.

Key words: Endometriosis; Ovarian function; Traditional Chinese Medicine

\section{INTRODUCTION}

Endometriosis is a common disease, and $80 \%$ of endometriosis patients are with ovarian endometrial cysts (EMA). Laparoscopic cystectomy is a preferred therapy by many gynecologists and considered the gold standard surgical treatment for EMA due to a low postoperative recurrence rate, low reoperation rate, and a high rate of natural pregnancy (Alborzi et al., 2004). However, given the wide range of applications of laparoscopic surgery in patients with ovarian cysts, decreased ovarian function after EMA surgery has garnered increasing attention from many scholars. One study by Benaglia et al. (2010) found that EMA causes diminished ovarian reserve and in some cases premature ovarian failure because having EMA introduces an inherent risk of invasion, extensive and severe pelvic adhesions, intraoperative bleeding, loss of both ovarian follicles and normal tissue, intraoperative bleeding, and electrical damage. Most EMA patients also develop fertility problems. Preserving the postoperative ovarian function of patients and improving their quality of life, in particular protecting the fertility of younger patients, remain important issues for gynecologists. The goal of the present study was to investigate changes in the postoperative ovarian reserve of patients who had undergone laparoscopic EMA cystectomy and to investigate how TCM optimized or improved ovarian reserve levels.

\section{MATERIAL AND METHODS}

\section{Subjects}

Seventy patients were enrolled and randomized into two groups between October 2009 and June 2012. EMA diagnoses were confirmed through laparoscopic examination by doctors in the Department of Gynecology, Guangdong Provincial Hospital of Chinese Medicine. Chinese dialectical examination was performed in eligible patients to determine the presence of kidney asthenia and blood stasis. Enrolled patients were randomly divided into two 
groups: one group receiving TCM $(\mathrm{N}=35)$ and a control group receiving usual care $(\mathrm{N}=35)$. The average age of patients in the study was 27.58 (range 21-36). No statistically significant differences in age, menstrual case, history of infertility, and endometrial clinical scores were documented among the two groups $(\mathrm{P}>0.05)$.

\section{Inclusion criteria}

Patients meeting the following inclusion criteria were eligible to participate in the study: 1) met the diagnostic criteria for Chinese and Western clinical medicine; 2) between $18-40$ years old at the time of study enrollment; 3 ) had received a surgical indication of EMA $\geq$ $5 \mathrm{~cm}$; 4) had not undergone hormone therapy for endometriosis three months preoperatively; 5) met diagnostic criteria confirmed through laparoscopy or histopathologic diagnosis; and 6) had completed informed consent for the study.

\section{Exclusion criteria}

Patients were excluded from participating in the study if they met any of the following exclusion criteria: 1) younger than 18 years or older than 40 at the time of study enrollment; 2) had received hormone therapy for endometriosis within three months preoperatively; 3 ) diagnosed with any other serious co-morbid condition including cardiovascular, liver, kidney or blood disease; 4) histopathologically diagnosed with other types of ovarian tumors or with EMA and other ovarian cancers; and 5) diagnosed with mental illness or deemed unable to take care of themselves.

\section{Treatment}

For the TCM group, laparoscopic EMA cystectomy was performed during the first half of the patient's menstrual cycle (prior to ovulation). After the first menstrual cycle, participants in this arm were prescribed TCM for kidney reinforcement and blood activation. The prescription was as follows: Semen Cuscutae (20 g), Herba Taxillus chinensis (20 g), Radix Dipsaci (15 g), Radix Angelicae sinensis (10 g), Radix Salviae miltiorrhizae (10 g), Radix Curcumae wenyujin (10 g), Rhizoma Cyperi (10 g), et al. The herbal medicines prescribed were pulverized into a fine powder. A daily dose was mixed thoroughly with $200 \mathrm{~mL}$ of warm water and taken orally by the patient once a day for three menstrual cycles, except during menses. For the control group, laparoscopic EMA myomectomy was performed during the first half of the menstrual cycle (prior to ovulation) and no TCM treatment was prescribed following surgery.

\section{Observation indexes}

Venous blood samples ( $3 \mathrm{~mL}$ to $5 \mathrm{~mL}$ ) samples were collected on days 2 and 3 of the surgical menstrual cycle and during menstrual cycles and during the first and fourth menstrual cycle post-operatively. Blood samples were centrifuged in order to collect serum, which was then stored at $-70^{\circ} \mathrm{C}$. Chemiluminescence was applied to detect levels of follicle stimulating hormone (FSH), luteinizing hormone (LH) and estradiol (E2) using corresponding kits (Siemens Medical Solutions Diagnostics, Inc., Chicago, USA). Serum INHB was assayed using an enzyme-linked immunosorbent assay (Beckman Coulter Co., Ltd., USA). Assays were performed in accordance with the manufacturer instructions. 


\section{Statistical analysis}

Statiscal analysis was performed using the SPSS 17.0 statistical software (version 17.0: SPSS, Chicago, IL, USA). The measurement data with normal distribution are reported as means $\pm \mathrm{SD}$. Differences between multiple groups were compared using a Mann-Whitney $\mathrm{U}$ test. $\mathrm{P}<0.05$ was considered to be statistically significant.

\section{RESULTS}

\section{Menstruation situations}

While the abnormal menstruation rates of both groups increased slightly during the 1 st month postoperatively, the difference was not statistically significant when compared with preoperative menstruation rates $(\mathrm{P}>0.05)$. The menstruation rates gradually returned to normal over time, as shown in Table 1.

\begin{tabular}{|c|c|c|c|c|c|c|c|}
\hline Group & Time & Cases & Normal & $\begin{array}{c}\text { Premature } \\
\text { menstruation }\end{array}$ & $\begin{array}{c}\text { Prolonged } \\
\text { menstruation }\end{array}$ & $\begin{array}{l}\text { More menstrual } \\
\text { blood volume }\end{array}$ & $\begin{array}{l}\text { Less menstrual } \\
\text { blood volume }\end{array}$ \\
\hline \multirow[t]{3}{*}{$\mathrm{TCM}$} & Preoperatively & 35 & 28 & 2 & 1 & 3 & 4 \\
\hline & Postoperative $1^{\text {st }}$ month & 35 & 23 & 5 & 4 & 1 & 7 \\
\hline & Postoperative $4^{\text {th }}$ month & 35 & 29 & 0 & 1 & 2 & 3 \\
\hline \multirow[t]{3}{*}{ Control } & Preoperatively & 35 & 29 & 3 & 2 & 3 & 2 \\
\hline & Preoperatively & 35 & 24 & 4 & 6 & 2 & 6 \\
\hline & Postoperative $4^{\text {th }}$ month & 35 & 25 & 3 & 6 & 3 & 5 \\
\hline
\end{tabular}

\section{Serum hormone and INHB levels}

Preoperative basal LH, FSH, E2, and INHB serum levels did not differ significantly between the two groups $(\mathrm{P}>0.05)$. At the 1st month postoperatively, the LH and FSH levels in both groups had increased, while the E2 and INHB levels had decreased in comparison to preoperative levels $(\mathrm{P}<0.05)$. After the 4th month, in comparison to preoperative levels, we observed a decrease in the LH and FSH levels of TCM group patients, yet their E2 and INHB levels had increased, although compared with the preoperative levels, the difference was not statistically significant $(\mathrm{P}>0.05)$. However, the difference in serum hormone levels measured during the 4th month differed significantly from those collected during the 1st month and from those in the control group $(\mathrm{P}<0.05)$, as shown in Table 2.

Table 2. Serum hormone and INHB level changes of the 2 groups before and after the treatment (means \pm SD).

\begin{tabular}{llccccc}
\hline Group & Time & Cases & FSH (IU/L) & LH (IU/L) & E2 (pM) & INHB (pg/mL) \\
\hline TCM & Preoperatively & 35 & $6.52 \pm 3.02$ & $5.87 \pm 2.82$ & $312.17 \pm 256.94$ & $67.78 \pm 11.78$ \\
& Postoperative 1st month & 35 & $11.10 \pm 5.84^{\Delta}$ & $9.72 \pm 4.68^{\Delta}$ & $214.40 \pm 155.38^{\Delta}$ & $43.30 \pm 12.24^{\Delta}$ \\
Control & Postoperative 4th month & 35 & $5.06 \pm 2.27^{* \#}$ & $5.45 \pm 5.19^{* \#}$ & $384.45 \pm 257.78^{* \#}$ & $64.50 \pm 16.56^{* \#}$ \\
& Preoperatively & 35 & $6.27 \pm 2.76$ & $5.73 \pm 3.74$ & $250.01 \pm 178.72$ & $68.80 \pm 12.69$ \\
& Postoperative 1st month & 35 & $10.96 \pm 6.78^{\Delta}$ & $9.51 \pm 8.02^{\Delta}$ & $159.78 \pm 154.53^{\Delta}$ & $48.91 \pm 13.25^{\Delta}$ \\
& Postoperative 4th month & 35 & $8.89 \pm 5.37^{\Delta}$ & $8.96 \pm 7.18^{\Delta}$ & $175.50 \pm 150.40^{\Delta}$ & $55.42 \pm 9.53^{\Delta}$ \\
\hline
\end{tabular}

${ }^{\Delta}$ Compared with the same group preoperatively, $\mathrm{P}<0.05$; *compared with the control group, $\mathrm{P}<0.05$; ${ }^{*}$ compared with the same group in the postoperative 1 st month, $\mathrm{P}<0.05$. 


\section{DISCUSSION}

Ovarian reserve refers to the capacity for follicle growth, development, and formation of a fertilized oocyte in the ovarian cortex (Bowan et al., 2007). When the ovarian reserve for egg production diminishes, oocyte quality also decreases. This results in a condition known as declined ovarian reserve or reduced fertility, which could eventually lead to ovarian failure. At present, the following indicators, markers, and tests are used to assess ovarian reserve: age, sex hormones (basal FSH, LH, and E2 levels), INHB, Mullerian duct antihormone levels, ovarian volume, and blood flow, clomiphene citrate challenge test (CCCT) and gonadotropin-releasing hormone agonist stimulation test (Vladimirov et al., 2005; Feyerisen et al., 2006; Dehghani-Firouzabadi et al., 2008; Busacca and Vignali, 2009).

Endometriosis of the ovary is a common site for endometriosis that can occur unilaterally or bilaterally. Laparoscopic surgery is currently the preferred mode of clinical treatment for the condition (Prospero and Micucci, 2009), particularly for younger patients who need to preserve their fertility. However, an increasing number of clinical studies have demonstrated that postoperative ovarian function is affected by ovarian endometriosis. This includes a reduction in the size of the residual ovary, changes in basic endocrine levels, diminished ovarian reserve, changes in ovulation function, and poor response to gonadotropin stimulation (Somigliana et al., 2003; Muzii et al., 2005; Donnez et al., 2010).

Compared with preoperative levels, this study revealed an increase during the first postoperative menstrual cycle in basal serum levels of FSH and LH, and a statistically significant decrease in INHB and basal E2 serum levels $(\mathrm{P}<0.05)$. Currently, basal FSH levels are the most commonly used indicator of ovarian reserve in clinical practice and is measured at days two and three of the menstrual cycle (Joiner et al., 2007; Singer et al., 2009). INHB is generated by the small and middle sinusoid ovarian follicles. Thus, the number of small and middle sinusoid ovarian follicles is positively correlated with basal INHB levels. The main physiologic function of INHB is to provide feedback inhibition of the pituitary FSH secretion by increasing E2 substrate generation and regulating E2 generation. Basal INHB levels are related to the ovarian reserve and are commonly used as laboratory evidence to predict clinical ovarian reserve (Corrine et al., 2005). Li et al (2009) found that, FSH levels increased after bilateral ovarian cystectomy, while the peak systolic velocity in ovarian stromal vessel antral follicles count and ovarian diameter decreased, with levels in the electrocoagulation group higher than in the suture group $(\mathrm{P}<0.05)$.

Several effects of surgery on ovarian function have been documented in the scientific literature. Studies have found that 1) electrocoagulation causes irreversible trauma to ovarian blood flow; 2) excessive removal of ovarian tissue effects ovarian function; and 3) severe local inflammation can trigger an auto-immune response in patients (Matsuzaki et al., 2009). In 2002, Hachisuga and Kawarabayashi found that stripping ovarian endometrial cysts can cause ovarian follicle loss and damage ovarian tissue. Garcia-Velasco et al. (2004) and Hart et al. (2008) also reached a similar conclusion. In a comparison of both ovaries, Somigliana et al. (2003) found that ovarian volume and the number of oocytes on the operated ovary was significantly more reduced than the other ovary that did not receive surgery. In addition, among the six research studies which reported ovulation induction therapy after ovarian endometriosis surgery, three studies mentioned that almost no mature follicles were generated ipsilateral to the operative site (Somigliana et al., 2006).

These results suggest that when TCM is administered to patients, postoperative FSH 
and LH levels can be reduced, while INHB and basic E2 levels increase, thereby invigorating the kidneys and activating the blood. These results confirm that certain TCM prescriptions improve the postoperative ovarian reserve of EMA patients. However, a larger sample size and rigorous prospective double-blind randomized studies are needed to validate these findings and provide stronger experimental evidence. Finally, TCM prescriptions that invigorate the kidney and activate the blood warrant further investigation.

\section{REFERENCES}

Alborzi S, Momtahan M, Parsanejhad ME, Dehbashi S, et al. (2004). A prospective, randomized study comparing laparoscopic ovarian cystectomy versus fenestration and coagulation in patients with endometriomas. Fertil. Steril. 82: 1633-1637.

Benaglia L, Somigliana E, Vighi V, Ragni G, et al. (2010). Rate of severe ovarian damage following surgery for endometriomas. Hum. Reprod. 25: 678-682.

Bowan S, Norian J, Santoro N and Pal L (2007). Simple tools for assessment of ovarian reserve (OR): individual ovarian dimensions are reliable predictors of OR. Fertil. Steril. 88: 390-395.

Busacca M and Vignali M (2009). Endometrioma excision and ovarian reserve a dangerous relation. J. Minim. Invas. Gynecol. 16: 142-148.

Corrine KW, Taylor AE, Fox J, Messerlian GM, et al. (2005). Follicular arrest in polycystic ovary syndrome is associated with deficient inhibin A and B biosynthesis. J. Clin. Endocr. Metab. 90: 5582-5587.

Dehghani-Firouzabadi R, Tayebi N and Asgharnia M (2008). Serum level of antimullerian hormone in early follicular phase as a predictor phase as a predictor of ovarian reserve and pregnancy outcome in assisted reproductive technology cycles. Arch. Iran. Med. 11: 371-376.

Donnez J, Lousse C, Jadoul P, Donnez O, et al. (2010). Laparoscopic management of endometriomas using a combined technique of excisional (cystectomy) and ablative surgery. Fertil. Steril. 94: 28-32.

Feyerisen E, Mendez Lozano DH, Taieb J, Hesters L, et al. (2006). Anti-Mullerian hormone: clinical insights into a promising biomarker of ovarian follicular status. Reprod. Biomed. Online 12: 695-703.

Garcia-Velasco JA, Mahutte NG, Corona J, Zúñiga V, et al. (2004). Removal of endometriomas before in vitro fertilization does not improve fertility outcomes: a matched, case-control study. Fertil. Steril. 81: 1194-1197.

Hachisuga T and Kawarabayashi T (2002). Histopathological analysis of laparoscopically treated ovarian endometriotic cysts with special reference to loss of follicles. Hum. Reprod. 17: 432-435.

Hart RJ, Hickey M, Maouris P and Buckett W (2008). Excisional surgery versus ablative surgery for ovarian endometriomata. Cochrane Database Syst. Rev. CD004992.

Joiner LL, Robinson RD, Bates W and Propst AM (2007). Establishing institutional critical values of follicle-stimulating hormone levels to predict in vitro fertilization success. Mil. Med. 172: 202-204.

Li CZ, Liu B, Wen ZQ and Sun Q (2009). The impact of electrocoagulation on ovarian reserve after laparoscopic excision of ovarian cysts: a prospective clinical study of 191 patients. Fertil. Steril. 92: 1428-1435.

Matsuzaki S, Houlle C, Darcha C, Pouly JL, et al. (2009). Analysis of risk factors for the removal of normal ovarian tissue during laparoscopic cystectomy for ovarian endometriosis. Hum. Reprod. 24: 1402-1406.

Muzii I, Bellati F, Bianchi A, Palaia I, et al. (2005). Laparoscopic stripping of endometriomas: a randomized trial on different surgical techniques. Part II: pathological results. Hum. Reprod. 20: 1987-1992.

Prospero D and Micucci K (2009). Is operative laparoscopy safe in ovarian endometriosis. Reprod. Biomed. Online 18: 167-169.

Singer T, Barad DH, Weghofer A and Gleicher N (2009). Correlation of antimullerian hormone and baseline folliclestimulating hormone levels. Fertil. Steril. 91: 2616-2619.

Somigliana E, Ragni G, Benedetti F, Borroni R, et al. (2003). Does laparoscopic excision of endometriotic ovarian cysts significantly affect ovarian reserve? Insights from IVF cycles. Hum. Reprod. 218: 2450-2453.

Somigliana E, Vercellini P, Viganó P, Ragni G, et al. (2006). Should endometriomas be treated before IVF-ICSI cycles? Hum. Reprod. Update 12: 57-64.

Vladimirov IK, Taeheva DM, Kalinov KB, Ivanova AV, et al. (2005). Prognostic value of some ovarian reserve tests in poor responders. Arch. Gynecol. Obstet. 272: 74-79. 